\title{
FINDING OF THE EXPECTED INCOME OF THE CLOSED QUEUEING STRUCTURE AND ITS APPLICATION IN TRANSPORT LOGISTICS
}

\author{
Mikhail Matalytski ${ }^{1}$, Olga Kiturko ${ }^{2}$ \\ ${ }^{I}$ Institute of Mathematics, Czestochowa University of Technology \\ Czestochowa, Poland \\ ${ }^{2}$ Faculty of Mathematics and Computer Science, Grodno State University \\ Grodno, Belarus \\ Im.matalytski@gmail.com, ${ }^{2}$ sytaya_om@mail.ru
}

\begin{abstract}
Research of the closed exponential queueing structure with one-type messages and the income is conducted. The differential equation in partial derivatives for an income distribution density is received. The ordinary differential equation for its expected income is constructed at particular starting conditions. The offered method of its decision in a case when the intensity of the service of messages, the number of messages in networks, the number of lines of the service in systems, the matrix of probabilities of transitions of messages and the income from transitions between conditions of the closed queueing structure (CQS) depend on time is described. An example when change of parameters has seasonal nature is reviewed. Results of this article can be applied at the prediction of the income of the logistic transport system (LTS).
\end{abstract}

\section{Introduction}

In LTS in practice, the total number of the vehicles moving between various objects and number of loading and unloading crews (workers or, for example, operating loading and unloading tracks at stations) depend on time. Therefore the following CQS can be considered as a LTS model.

Let's consider the closed queueing network consisting of $n+1$ queueing systems $S_{0}, S_{1}, \ldots, S_{n}$, the total number of one-type messages in which in an instant $t$ makes $K(t)$. Usually the system $S_{0}$ is understood as environment, and as systems $S_{1}, S_{2}, \ldots, S_{n}$ - concrete queueing systems of network in which the service of messages is made. The queueing networks closed on structure but having not constant total number of served messages, depending on time, are investigated for the first time in special cases in [1] and called the closed queueing structures (CQS). Let $m_{i}(t)$ - number of service lines in system $S_{i}, i=\overline{1, n}$, we will put $m_{0}(t)=K(t)$, and times of messages service of each of service lines are distributed under the exponential law with intensity $\mu_{i}(t)$, also time-dependent, $i, j=\overline{0, n}$. 
Messages for the service get out according to discipline of FIFO. The message, whose service in system $S_{i}$ ended, with probability $p_{i j}(t)$ passes to system $S_{j}$, $i, j=\overline{0, n}$. The matrix of transition probabilities $P(t)=\left\|p_{i j}(t)\right\|, i, j=\overline{0, n}$, is a matrix of transition probabilities of a nonreducible Markov chain in each moment $0 \leq p_{i j}(t) \leq 1, \sum_{j=0}^{n} p_{i j}(t)=1$. The primal problem of research of the given CQS consists in the asymptotic analysis of the Markov process describing its behavior at a large number of messages.

The condition of structure in a moment $t$ is described by a vector

$$
k(t)=(k, t)=\left(k_{1}, k_{2}, \ldots, k_{n}, t\right)=\left(k_{1}(t), k_{2}(t), \ldots, k_{n}(t)\right)
$$

where $k_{i}(t)$ - number of messages in system $S_{i}$ in a moment $t, t \in[0, T], i=\overline{0, n}$, which forms the $n$-dimensional Markov process with the continuous time and a finite number of conditions. It is apparent that the number of messages in system $S_{0}$ is equal to $k_{0}(t)=K(t)-\sum_{i=1}^{n} k_{i}(t)$.

Believing that transitions of messages between systems bring in to CQS the particular income, we will set the task of prediction of its total expected income. Let $V(k, t)$ - the complete expected income which will receive CQS in time $t$ if in an initial moment it is in a condition $(k, t)$. It is apparent that $V(k, t)=\sum_{i=0}^{n} V_{i}(k, t)$, where $V_{i}(k, t)$ - the expected income which is gained by system $S_{i}$ in time $t$ if in an initial moment the CQS is in a condition $k$. In article [2], the concept of distribution density of the expected income of CQS $v(x, t), x=\left(x_{1}, x_{2}, \ldots, x_{n}\right)$ is injected, and the following statement is proved.

Theorem. Income distribution density $v(x, t)$ under a condition that it is differentiated on $t$ and is twice sectionally continuous differentiated on $x_{i}, i=\overline{1, n}$, satisfies the terms of order of smallness $\varepsilon^{2}(t)=\frac{1}{K^{2}(t)}$ to the following differential equation in partial derivatives:

$$
\begin{gathered}
\frac{\partial v(x, t)}{\partial t}=-\sum_{i=1}^{n} A_{i}(x, t) \frac{\partial v(x, t)}{\partial x_{i}}-\frac{\varepsilon(t)}{2} \sum_{i, j=1}^{n} B_{i j}(x, t) \frac{\partial^{2} v(x, t)}{\partial x_{i} \partial x_{j}}- \\
-n K(t) \varepsilon^{\prime}(t) v(x, t)+r(t)+K(t) \sum_{i, j=0}^{n} \mu_{j}(t) p_{j i}(t) \min \left(l_{j}(t), x_{j}\right) r_{j i}(t)
\end{gathered}
$$


where

$$
\begin{gathered}
A_{i}(x, t)=\sum_{j=1}^{n} \mu_{j}(t) p_{j i}^{*}(t) \min \left(l_{j}(t), x_{j}\right)+\mu_{0}(t) p_{0 i}(t)\left(1-\sum_{i=1}^{n} x_{i}\right), \\
p_{j i}^{*}(t)=\left\{\begin{array}{l}
p_{j i}(t)-1, j=i, \\
p_{j i}(t), j \neq i,
\end{array} l_{j}(t)=\frac{m_{j}(t)}{K(t)}, j=\overline{1, n},\right.
\end{gathered}
$$

$B_{i j}(x, t)$ - sectionally continuous function concerning $x$,

$$
\begin{gathered}
B_{i i}(x, t)=\sum_{j=0}^{n} \mu_{j}(t) q_{j i}^{*}(t) \min \left(l_{j}(t), x_{j}\right), B_{i j}(x, t)=\mu_{i}(t) p_{i j}(t) \min \left(l_{i}(t), x_{i}\right), \\
q_{j i}^{*}(t)=\left\{\begin{array}{l}
-1-p_{j i}(t), j=i, \\
-p_{j i}(t), j \neq i .
\end{array}\right.
\end{gathered}
$$

\section{Differential equation for the average income of structure}

Considering (3), expression $\frac{\varepsilon(t)}{2} \sum_{i, j=1}^{n} B_{i j}(x, t) \frac{\partial^{2} v(x, t)}{\partial x_{i} \partial x_{j}}$ can be referred to $O\left(\varepsilon^{2}(t)\right)$. Therefore we will consider the following equation:

$$
\begin{aligned}
& \frac{\partial v(k, t)}{\partial t}=-\sum_{i=1}^{n} A_{i}(x, t) \frac{\partial v(x, t)}{\partial x_{i}}-n K(t) \varepsilon^{\prime}(t) v(x, t)+ \\
& +K(t) \sum_{i, j=0}^{n} \mu_{j}(t) p_{j i}(t) \min \left(l_{j}(t), x_{j}\right) r_{j i}(t)+r(t) .
\end{aligned}
$$

Having integrated both parts of this equation on $x=\left(x_{1}, x_{2}, \ldots, x_{n}\right)$ in area $G=\left\{x=\left(x_{1}, x_{2}, \ldots, x_{n}\right): x_{i} \geq 0, \sum_{i=1}^{n} x_{i} \leq 1\right\}$ and having divided both members of equation into the volume of area $G$, equal to $m(G)$, we receive:

$$
\begin{gathered}
\frac{1}{m(G)} \iint_{G} \ldots \int \frac{\partial v(x, t)}{\partial t} d x=-\frac{1}{m(G)} \sum_{i=1}^{n} \iint_{G} \ldots \int A_{i}(x, t) \frac{\partial v(x, t)}{\partial x_{i}} d x- \\
-\frac{n K(t) \varepsilon^{\prime}(t)}{m(G)} \iint_{G} \ldots \int v(x, t) d x+\frac{K(t)}{m(G)} \sum_{\substack{i=0, j=1}}^{n} \mu_{j}(t) p_{j i}(t) r_{j i}(t) \iint_{G} \ldots \int \min \left(l_{j}(t), x_{j}\right) d x+ \\
+\frac{K(t)}{m(G)} \sum_{i=0}^{n} \mu_{0}(t) p_{0 i}(t) r_{0 i}(t) \iint_{G} \ldots \int\left(1-\sum_{j=1}^{n} x_{j}\right) d x+\frac{r(t)}{m(G)} \iint_{G} \ldots \int d x
\end{gathered}
$$


Let's consider that in the left part of this equality the change of order of an integration and derivation (we assume that in closed area $G$ function $v(x, t)$ is the continuous) is admissible:

$$
\frac{1}{m(G)} \iint_{G} \ldots \int \frac{\partial v(x, t)}{\partial t} d x=\frac{1}{m(G)} \frac{\partial}{\partial t} \iint_{G} \ldots \int v(x, t) d x=\frac{d}{d t} \overline{v_{G}}(t),
$$

where $\overline{v_{G}}(t)$ - an average on $x$ value of the income on condition of change of a reference state $(x, t)$ in area $G$.

Let's consider integrals in a right member (4). It is apparent that $\frac{n K(t) \varepsilon^{\prime}(t)}{m(G)} \iint_{G} \ldots \int v(x, t) d x=n K(t) \varepsilon^{\prime}(t) \overline{v_{G}}(t)$. During calculation of the remaining integrals we use integration by parts, and also we will assume that boundary conditions [3] are satisfied:

$$
\left.A_{i}(x, t) v(x, t)\right|_{x \in \Gamma(G)}=0, i=\overline{1, n},
$$

were area border $\quad G$, i.e. $\left.\quad A_{n}(x, t) v(x, t)\right|_{x_{n}=0} ^{x_{n}=1-x_{1}-x_{2}-\ldots-x_{n-1}}=0$, $\left.A_{n-1}(x, t) v(x, t)\right|_{x_{n-1}=0} ^{x_{n-1}=-x_{1}-x_{2}-\ldots-x_{n-2}-x_{n}}=0, \ldots,\left.A_{1}(x, t) v(x, t)\right|_{x_{1}=0} ^{x_{1}=1-x_{2}-x_{3}-\ldots-x_{n}}=0$, which means that the flow of the income through the border of area $G$ is not assumed or that in boundary points of area $G$ reflecting screens are applied. Then, considering that $\frac{\partial A_{i}(x, t)}{\partial x_{i}}$ does not depend on $x_{j}, j=\overline{1, n}$, we receive

$$
\frac{1}{m(G)} \iint_{G} \ldots \int A_{i}(x, t) \frac{\partial v(x, t)}{\partial x_{i}} d x=-\frac{\partial A_{i}(x, t)}{\partial x_{i}} \overline{v_{G}}(t), i=\overline{1, n} .
$$

Therefore we come to the following differential equation

$$
\begin{gathered}
\frac{d}{d t} \overline{v_{G}}(t)=\left[\sum_{i=1}^{n} \frac{\partial A_{i}(x, t)}{\partial x_{i}}-n K(t) \varepsilon^{\prime}(t)\right] \overline{v_{G}}(t)+ \\
+\frac{K(t)}{m(G)} \sum_{j=1}^{n} \sum_{i=0}^{n} \mu_{j}(t) p_{j i}(t) r_{j i}(t) \iint_{G} \ldots \int \min \left(l_{j}(t), x_{j}\right) d x+ \\
+\frac{K(t)}{m(G)} \sum_{i=0}^{n} \mu_{0}(t) p_{0 i}(t) r_{0 i}(t) \iint_{G} \ldots \int\left(1-\sum_{j=1}^{n} x_{j}\right) d x+\frac{r(t)}{m(G)} \iint \ldots \int d x .
\end{gathered}
$$

From (2) we see that the coefficients $A_{i}(x, t)$ represent piecewise linear functions on $x_{j}, j=\overline{1, n}$, that is, (5) is a differential equation with a piecewise constant right member. Let's designate a set of indexes of components of a vector 
$x=\left(x_{1}, x_{2}, \ldots, x_{n}\right)$ through $\Omega=\{1,2, \ldots, n\}$. Let's break $\Omega$ into two non-overlapping sets $\Omega_{0}(\tau), \Omega_{1}(\tau)$ so that $\Omega_{0}(\tau)=\left\{j: l_{j}(t)<x_{j} \leq 1\right\}, \Omega_{1}(\tau)=\left\{j: 0 \leq x_{j} \leq l_{j}(t)\right\}$, $\tau$ - splitting number. At fixed $t$, the number of splittings of this kind is equal to $2^{n}, \tau=\overline{1,2^{n}}$. Each splitting will set in a set $G$ not being crossed areas $G_{\tau}$ so that

$$
\begin{gathered}
G_{\tau}=\left\{x(t): l_{i}(t)<x_{i} \leq 1, i \in \Omega_{0}(\tau) ; 0 \leq x_{j} \leq l_{j}(t), j \in \Omega_{1}(\tau) ; \sum_{i=1}^{n} x_{i} \leq 1\right\}, \\
\tau=1,2, \ldots, 2^{n}, \bigcup_{\tau=1}^{2^{n}} G_{\tau}=G .
\end{gathered}
$$

Now in each area splitting of a phase space we can write down an apparent look (5) and at particular starting conditions find the average expected income for each of the areas $G_{\tau}$.

Let's set, for example, splitting: $\Omega_{0}(1)=\{1,2, \ldots, n\}, \Omega_{1}(1)=\{\emptyset\}, \tau=1$, that corresponds to existence of queues $S_{1}, S_{2}, \ldots, S_{n}$. Then, solving the equation (5) at starting conditions $\overline{v_{G_{1}}}(0)=S$, it is possible to determine the average expected income changing of a reference state of area $G_{1}=\left\{x(t): l_{i}(t)<x_{i} \leq 1, i=\overline{1, n}, \sum_{i=1}^{n} x_{i} \leq 1\right\}$. The equation (5) thus looks like

$$
\begin{gathered}
\frac{d}{d t} \overline{v_{G_{1}}}(t)=\left[\mu_{0}(t) \sum_{i=1}^{n} p_{0 i}(t)-n K(t) \varepsilon^{\prime}(t)\right] \overline{v_{G_{1}}}(t)+ \\
+\frac{1}{m\left(G_{1}\right)}\left[\sum_{j=1}^{n} \sum_{i=0}^{n} \mu_{j}(t) p_{j i}(t) m_{j}(t) r_{j i}(t)+\right. \\
\left.+K(t) \sum_{i=0}^{n} \mu_{0}(t) p_{0 i}(t) r_{0 i}(t) \iiint_{G_{1}} \ldots \int\left(1-\sum_{j=1}^{n} x_{j}\right) d x\right]+r(t) .
\end{gathered}
$$

\section{Example}

The transport enterprise (TE, system $S_{3}$ ) at the disposal of which a large number of cars (messages) is available, sends the cars for realization of a number of particular transportations between various cities (environment, system $S_{0}$ ). After that they come back to the TE base, before having passed in two points (systems $S_{1}$ and $S_{2}$ ) technical inspection which can also include car repairs. The number of functioning lines of a service in points $S_{1}$ and $S_{2}$ in a moment $t$ are equal respec- 
tively to $m_{1}(t)$ and $m_{2}(t)$. In system $S_{3}$ loading of cars before the flight in which are engaged $m_{3}(t)$ loading crews (service lines) is carried out.

A similar situation can arise when cars come back from the environment and are unloaded in two warehouses TE (systems $S_{1}$ and $S_{2}$ ); in this case $m_{1}(t)$ and $m_{2}(t)$ - number of crews of unloading accordingly in the warehouse $S_{1}$ and $S_{2}$.

In both cases the model of functioning of transportations is CQS represented in Figure 1.

It is apparent that in this case the matrix $P(t)$ looks like

$$
P(t)=\left\|p_{i j}(t)\right\|_{4 \times 4}=\left(\begin{array}{cccc}
0 & p_{01}(t) & p_{02}(t) & 0 \\
0 & 0 & 0 & 1 \\
0 & 0 & 0 & 1 \\
1 & 0 & 0 & 0
\end{array}\right), p_{01}(t)+p_{02}(t)=1, \forall t \in[0, T] .
$$

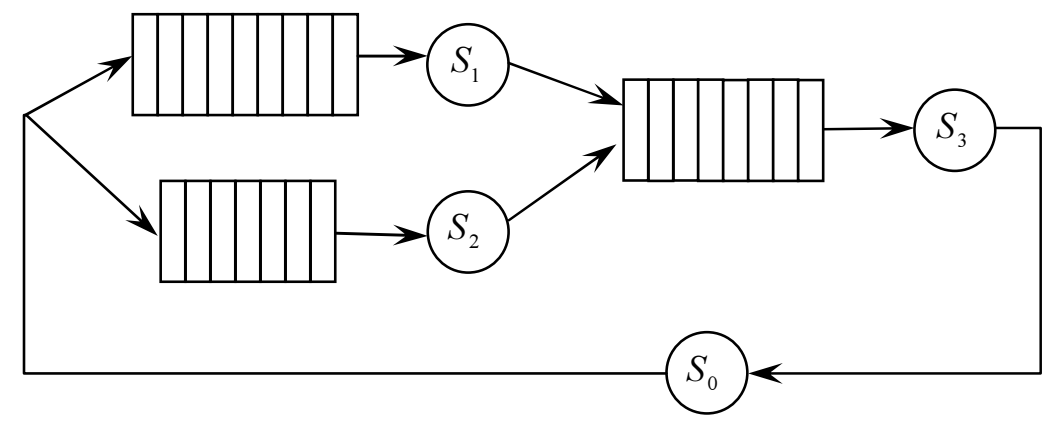

Fig. 1. Model of cars, movement

It is apparent that in this case the matrix looks like

$$
P(t)=\left\|p_{i j}(t)\right\|_{4 \times 4}=\left(\begin{array}{cccc}
0 & p_{01}(t) & p_{02}(t) & 0 \\
0 & 0 & 0 & 1 \\
0 & 0 & 0 & 1 \\
1 & 0 & 0 & 0
\end{array}\right), p_{01}(t)+p_{02}(t)=1, \forall t \in[0, T] .
$$

Let the change of the CQS parameters have a seasonal nature, for example, they can have one value during the winter period and others in the summer. Let's establish that

$$
K(t)=\left\{\begin{array}{l}
K_{1}, t \in\left[0, \frac{T}{2}\right), \\
K_{2}, t \in\left[\frac{T}{2}, T\right],
\end{array} \quad \mu_{j}(t)=\left\{\begin{array}{l}
\mu_{j}^{(1)}, t \in\left[0, \frac{T}{2}\right), \\
\mu_{j}^{(2)}, t \in\left[\frac{T}{2}, T\right]
\end{array} \quad j=\overline{0,3},\right.\right.
$$




$$
m_{j}(t)=\left\{\begin{array}{l}
m_{j}^{(1)}, t \in\left[0, \frac{T}{2}\right), \\
m_{j}^{(2)}, t \in\left[\frac{T}{2}, T\right],
\end{array} \quad j=\overline{1,3}, p_{0 i}(t)=\left\{\begin{array}{l}
p_{0 i}^{(1)}, t \in\left[0, \frac{T}{2}\right), \\
p_{0 i}^{(2)}, t \in\left[\frac{T}{2}, T\right]
\end{array} \quad i=1,2 .\right.\right.
$$

Also let the income from transitions between conditions of CQS also be step functions with two intervals of constancy:

$$
r_{j i}(t)=\left\{\begin{array}{l}
r_{j i}^{(1)}, t \in\left[0, \frac{T}{2}\right), \\
r_{j i}^{(2)}, t \in\left[\frac{T}{2}, T\right],
\end{array} \quad i, j=\overline{0,3}, r(t)=\left\{\begin{array}{l}
r^{(1)}, t \in\left[0, \frac{T}{2}\right), \\
r^{(2)}, t \in\left[\frac{T}{2}, T\right] .
\end{array}\right.\right.
$$

It is clear that $\varepsilon^{\prime}(t)=0$.

Let's find area volume $G_{1}=\left\{x: l_{i}<x_{i} \leq 1, i \in\{1,2,3\} ; 0 \leq x_{0} \leq l_{0} ; \sum_{i=1}^{3} x_{i} \leq 1\right\}$. We have

$$
m\left(G_{1}\right)=\iiint_{G_{1}} d x=\int_{l_{1}}^{1-l_{2}} d x_{1} \int_{l_{2}}^{1-x_{1}} d x_{2} \int_{l_{3}}^{1-x_{1}-x_{2}} d x_{3}=\frac{\left(1-l_{1}-l_{2}\right)^{2}\left(1-l_{1}-l_{2}-3 l_{3}\right)}{6} .
$$

It is possible also to find integral $\iiint_{G_{1}}\left(1-x_{1}-x_{2}-x_{3}\right) d x$ :

$$
\begin{gathered}
\iiint_{G_{1}}\left(1-x_{1}-x_{2}-x_{3}\right) d x=\int_{l_{1}}^{1-l_{2}} d x_{1} \int_{l_{2}}^{1-x_{1}} d x_{2} \int_{l_{3}}^{1-x_{1}-x_{2}}\left(1-x_{1}-x_{2}-x_{3}\right) d x_{3}= \\
=\frac{\left(1-l_{1}-l_{2}\right)^{2}\left(\left(1-l_{1}-l_{2}\right)^{2}-4\left(1-l_{1}-l_{2}\right) l_{3}+6 l_{3}^{2}\right)}{24} .
\end{gathered}
$$

Let's first find the expected income of CQS $\overline{v_{1}}(t)$ on an interval $\left[0, \frac{T}{2}\right)$. The equation (6) in this case looks like:

$$
\begin{gathered}
\frac{d \overline{v_{1}}(t)}{d t}=\mu_{0}^{(1)} \overline{v_{1}}(t)+\frac{1}{m\left(G_{1}\right)}\left[\mu_{1}^{(1)} m_{1}^{(1)} r_{13}^{(1)}+\mu_{2}^{(1)} m_{2}^{(1)} r_{23}^{(1)}+\mu_{3}^{(1)} m_{3}^{(1)} r_{30}^{(1)}+\right. \\
\left.+K_{1} \mu_{0}^{(1)}\left(p_{01}^{(1)} r_{01}^{(1)}+p_{02}^{(1)} r_{02}^{(1)}\right) \iiint_{G_{1}}\left(1-x_{1}-x_{2}-x_{3}\right) d x+r^{(1)}\right]=\mu_{0}^{(1)} \overline{v_{1}}(t)+r_{1} .
\end{gathered}
$$


And let $\overline{v_{1}}(0)=v_{0}^{(1)}$. Its decision is

$$
\begin{gathered}
\overline{v_{1}}(t)=v_{0}^{(1)} e^{\mu_{0}^{(1) t}}+r_{1} \int_{0}^{t} e^{\mu_{0}^{(1)}(t-\tau)} d \tau=v_{0}^{(1)} e^{\mu_{0}^{(1)} t}+\frac{r_{1}}{\mu_{0}^{(1)}}\left(e^{\mu_{0}^{(1)} t}-1\right)= \\
=\left(v_{0}^{(1)}+\frac{r_{1}}{\mu_{0}^{(1)}}\right) e^{\mu_{0}^{(1)} t}-\frac{r_{1}}{\mu_{0}^{(1)}}, t \in\left[0, \frac{T}{2}\right) .
\end{gathered}
$$

Let's now find the expected income $\overline{v_{2}}(t)$ of CQS on interval $\left[\frac{T}{2}, T\right]$. The equation (6) on this interval becomes

$$
\frac{d \overline{v_{2}}(t)}{d t}=\mu_{0}^{(2)} \overline{v_{2}}(t)+r_{2}
$$

where $\quad r_{2}=\frac{1}{m\left(G_{1}\right)}\left[\mu_{1}^{(2)} m_{1}^{(2)} r_{13}^{(2)}+\mu_{2}^{(2)} m_{2}^{(2)} r_{23}^{(2)}+\mu_{3}^{(2)} m_{3}^{(2)} r_{30}^{(2)}+K_{2} \mu_{0}^{(2)}\left(p_{01}^{(2)} r_{01}^{(2)}+p_{02}^{(2)} r_{02}^{(2)}\right) \times\right.$ $\left.\times \iiint_{G_{1}}\left(1-x_{1}-x_{2}-x_{3}\right) d x+r^{(2)}\right]$, as starting conditions it is necessary to take $\overline{v_{2}}\left(\frac{T}{2}\right)=\overline{v_{1}}\left(\frac{T}{2}\right)$. The solution of the equation (7) is

$$
\overline{v_{2}}(t)=\left[\left(v_{0}^{(1)}+\frac{r_{1}}{\mu_{0}^{(1)}}\right) e^{\mu_{0}^{(1)} \frac{T}{2}}-\frac{r_{1}}{\mu_{0}^{(1)}}+\frac{r_{2}}{\mu_{0}^{(2)}}\right] e^{\mu_{0}^{(2)} t}-\frac{r_{2}}{\mu_{0}^{(2)}}, t \in\left[\frac{T}{2}, T\right] .
$$

\section{References}

[1] Matalytski M., Rusilko T., Mathematical analysis of stochastic models for claim processing in insurance companies, GrSU, Grodno 2007 (in Russian).

[2] Kiturko O., Matalytski M., Rusilko T., Asymptotic analysis of the total expected income of closed queueing structure with the one-type messages and application, Wiestnik GrUP 2013, 1(2) (in Russian).

[3] Tikhonov V., Mironov V., Markov processes, Sov. Radio, Moscow 1977 (in Russian). 